\title{
Target-mass dependence of the break-up of halo nuclei
}

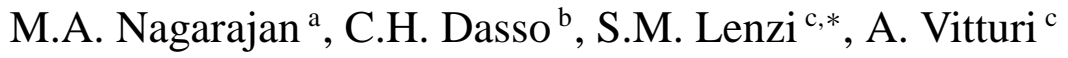 \\ a Department of Physics, UMIST, Manchester M60 1QD, UK \\ b Departamento de Física Atómica, Molecular y Nuclear, Universidad de Sevilla, Apdo. 1065, 41080 Sevilla, Spain \\ ${ }^{\mathrm{c}}$ Dipartimento di Fisica, Università di Padova and INFN, Via Marzolo 8, I-35131 Padova, Italy
}

Received 14 July 2000; received in revised form 20 December 2000; accepted 24 January 2001

Editor: J.-P. Blaizot

\begin{abstract}
Nuclear dissociation cross sections for halo nuclei have been assumed by various authors to have a $A^{1 / 3}$ dependence on the target mass. These cross sections have also been considered to be a sum of the nuclear and Coulomb components. Explicit calculations of the break-up of ${ }^{19} \mathrm{C}$ induced by various targets show that both of the above assumptions become invalid for halo nuclei. () 2001 Published by Elsevier Science B.V.
\end{abstract}

PACS: 21.60.-n; 23.20.Js

During recent years, the break-up of exotic halo nuclei has been investigated both theoretically and experimentally by several groups [1-6]. One of the main motivations of these studies is the possibility of determining, in selected cases, the astrophysical $\mathrm{S}$-factor. In fact, if the predominant contributions to the dissociation mechanism were due to first-order electromagnetic transitions into the continuum, the observed cross section could be related to those of the inverse photo-capture reaction process.

To apply this procedure, however, it is necessary to justify the absence of a significant nuclear contribution to the relevant excitation amplitudes. To this end it has been argued that the Coulomb and nuclear break-up processes are associated with rather distinct regions of the impact parameter range. In such case Coulombnuclear interference needs not be considered and the total break-up cross section would simply be the sum

\footnotetext{
* Corresponding author.

E-mail address: silvia.lenzi@pd.infn.it (S.M. Lenzi).
}

of those associated with the individual processes. According to this picture, Coulomb-induced break-up emerges, naturally, from the large impact parameters. Nuclear dissociation results, on the other hand, from a narrow band of grazing partial waves which - at very high bombarding energies - corresponds to impact parameters close to the sum of the radii of the colliding nuclei [7]. Note that this last sentence immediately suggests that the nuclear break-up cross sections should follow a $A^{1 / 3}$-dependence on the target mass (which is also the prediction of the Serber model [8], based on purely geometrical arguments). Such a scaling law has been invoked in halo-nuclei to establish a connection with the (small) nuclear break-up cross sections measured for stable systems $[9,10]$. Thus, "pure" Coulomb break-up cross sections have been obtained after the subtraction on the estimated nuclear contribution. Of particular interest is the case of the nucleus ${ }^{19} \mathrm{C}$, which is so far the heaviest halo nucleus experimentally studied [10]. The determination of the ground state configuration and neutron separation en- 
ergy have been recently the subject of several theoretical investigations. Most of their conclusions have been based on the comparison with the scaled experimental cross section $[11,12]$.

In this Letter we show that these considerations are not valid when applied to the break-up of halo nuclei. In fact, it is known that the transition densities of weakly-bound systems extend to unusually large radial distances [4,13-15]. One no longer enjoys a clear separation between the ranges of impact parameters leading to either Coulomb or nuclear dissociation and, consequently, Coulomb-nuclear interference inevitably plays an important role. To illustrate this problem and to investigate the dependence of the nuclear break-up cross sections of halo nuclei on the target mass, we have performed calculations for the break-up of ${ }^{19} \mathrm{C}$ induced by three different targets $\left({ }^{12} \mathrm{C},{ }^{58} \mathrm{Ni}\right.$ and $\left.{ }^{208} \mathrm{~Pb}\right)$ at $E / A=67 \mathrm{MeV}$.

To recreate the conditions of a normal, stable projectile, we first place the last neutron of ${ }^{19} \mathrm{C}$ in a $2 s_{1 / 2}$ state with an artificially large binding energy of $10 \mathrm{MeV}$. The single-particle wave function is obtained as an eigenstate of a Woods-Saxon potential whose depth is adjusted to yield the assumed binding energy. The neutron continuum states are calculated with the same potential. Projectile break-up is treated as an inelastic transition to a discretized set of continuum states, ignoring higher-order continuumcontinuum couplings which are expected to diminish their importance at high bombarding energy [16]. The details of the evaluation of the Coulomb and nuclear form factors and that of the break-up amplitudes have been previously summarized in Ref. [14]. For the ionion optical potential the parametrization of AkyuzWinther [17] has been used for the real part, while for the imaginary part we have used the same geometry and half its strength. The sensitivity of the results to the choice of the radius of the absorptive potential has been discussed in Ref. [14] To illustrate our point in a simple case, we consider here only dipole transitions for both the Coulomb and nuclear break-up processes, as they are considered to be predominant in this process. We have included in the calculation transitions to a bound $1 p_{1 / 2}$ state whose binding energy has been chosen to be $3 \mathrm{MeV}$.

In the left-upper panel of Fig. 1 we show, for the three targets, the dependence of the differential nuclear break-up cross section $d \sigma / d b$ as a function of the impact parameter $b$. Note that the total nuclear cross sections, whose value is indicated close to each curve, roughly scale with the target mass as $A^{1 / 3}$. The other figures of the left panel of Fig. 1 show that, although the Coulomb and nuclear contributions originate in different regions of the impact parameter (the nuclear being peaked at the grazing and the Coulomb extending smoothly to large impact parameters) there is an appreciable effect of interference.

We now move to the realistic case of ${ }^{19} \mathrm{C}$ where the last neutron has been estimated to be bound by only $530 \mathrm{keV}$ [10]. We have considered in the calculations transitions to $\mathrm{p}_{1 / 2}$ continuum states, but similar qualitative features apply when also the transitions to the $\mathrm{p}_{3 / 2}$ states are included. We first consider the case of $\mathrm{a}^{208} \mathrm{~Pb}$ target. The nuclear and Coulomb form factors are shown in Fig. 2 for the coupling to $\mathrm{p}_{1 / 2}$ states at a continuum energy of $\approx 0.35 \mathrm{MeV}$, which corresponds to the peak in the $Q$-distribution. As anticipated, the nuclear form factor now extends to large distances and cannot be neglected in comparison to the Coulomb form factor up to around $25 \mathrm{fm}$. In Fig. 3 the continuum energy dependence of the nuclear and Coulomb form factors are shown for a fixed distance of $20 \mathrm{fm}$. Even at this large distance, both form factors are comparable in magnitude. Thus, one would expect considerable nuclear-Coulomb interference over a large range of impact parameters. This is seen in Fig. 4, where the differential break-up cross sections $d \sigma / d b$ are shown as a function of the impact parameter. It is clear that one has to go much beyond $25 \mathrm{fm}$ in impact parameter before the cross section is well described as a pure Coulomb dissociation process (this would correspond to very small forward angles in the angular distribution). In the rest of the impact parameter range there is clear evidence of nuclear-Coulomb interference. This is also seen in Fig. 5a, where we show the differential break-up cross sections as a function of the continuum energy, integrated over all impact parameters. The break-up cross section clearly shows the presence of a large interference between the nuclear and Coulomb contributions. Note that the Coulomb and nuclear break-up amplitudes are in phase in the case of ${ }^{19} \mathrm{C}$ because the neutron halo has an effective negative charge. This feature persists even if one restricts the analysis to the most forward-angles. As an example, in Fig. 5b we consider only the contribution to the cross sections from impact parameters larger 

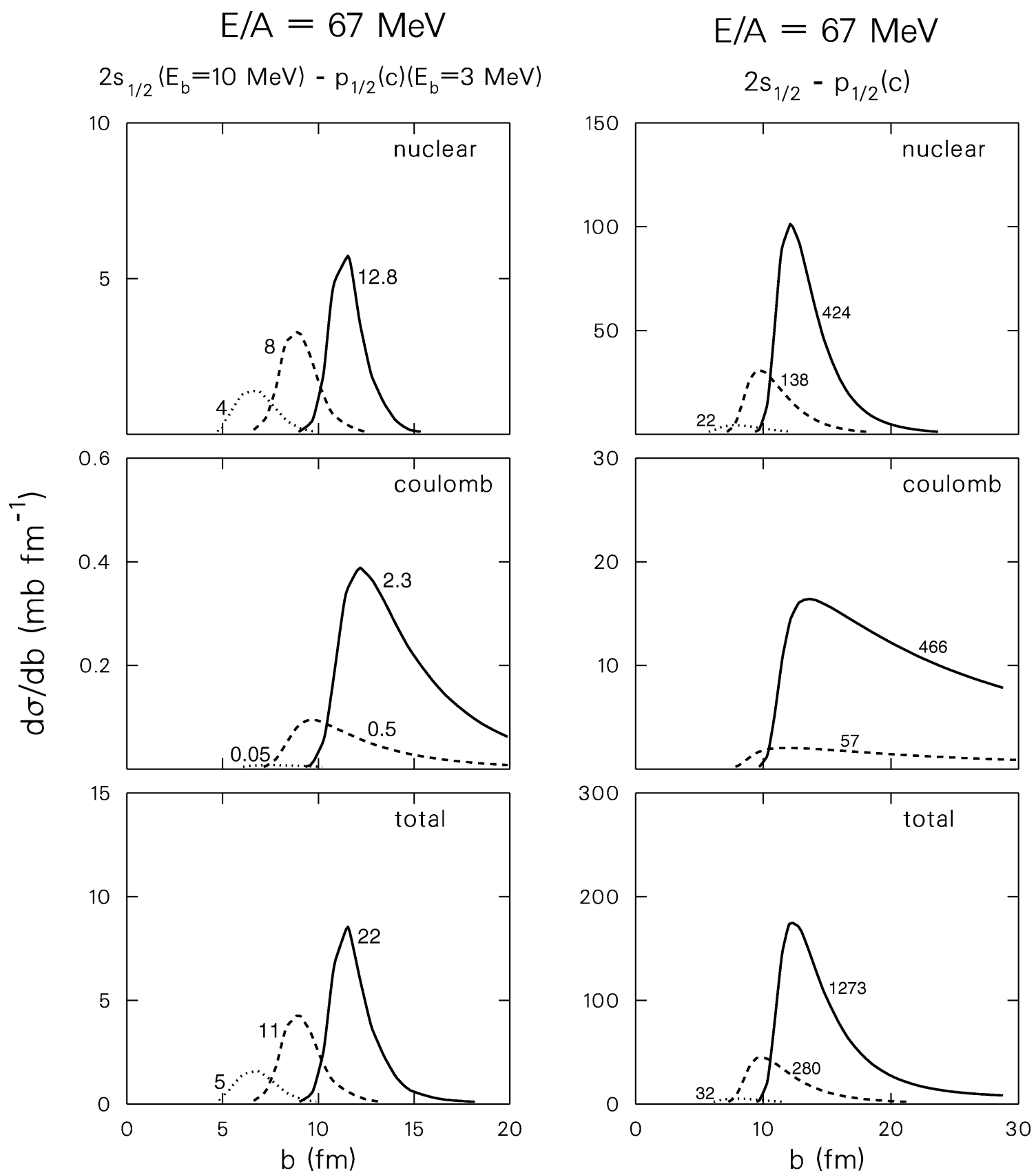

Fig. 1. Differential break-up cross sections as a function of the impact parameter. The three curves in each frame refer to the break-up of ${ }^{19} \mathrm{C}$ on ${ }^{12} \mathrm{C}$ (dotted), ${ }^{58} \mathrm{Ni}$ (dashed) and ${ }^{208} \mathrm{~Pb}$ targets (solid), at a bombarding energy of $E / A=67 \mathrm{MeV}$. In the left panel the considered transition is from a well bound $\left(E_{B}=10 \mathrm{MeV}\right) 2 \mathrm{~s}_{1 / 2}$ state in ${ }^{19} \mathrm{C}$ to a bound $1 \mathrm{p}_{1 / 2}$ state, while in the right panel, we consider the realistic case of a transition from a weakly bound $\left(E_{B}=530 \mathrm{keV}\right) 2 \mathrm{~s}_{1 / 2}$ state to all integrated $\mathrm{p}_{1 / 2}$ states in the continuum. The different frames refer to the cases in which either the nuclear, the Coulomb break-up, or both are considered. Values of the total cross sections are indicated close to each curve. 


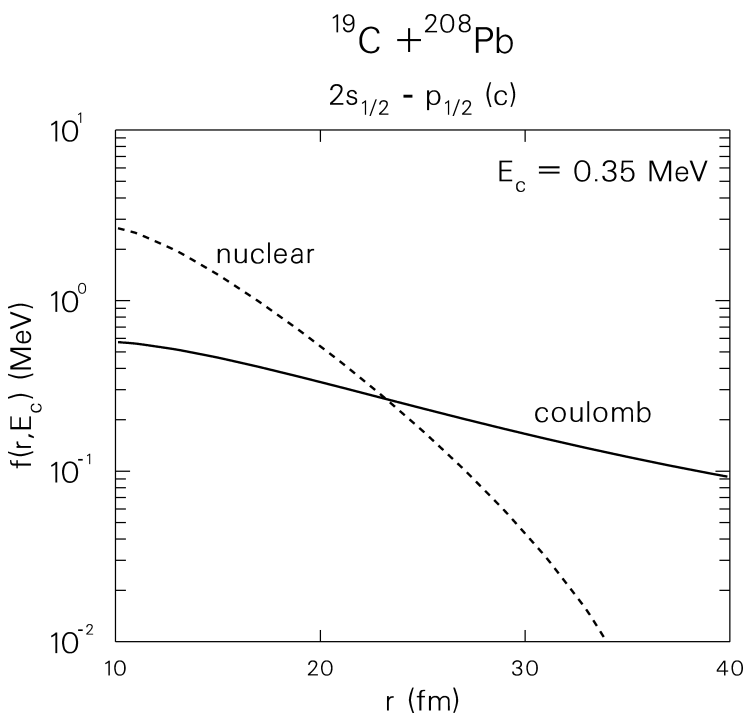

Fig. 2. Radial dependence of the nuclear and Coulomb form factors for the coupling from the bound $2 s_{1 / 2}$ state in ${ }^{19} \mathrm{C}\left(E_{b}=530 \mathrm{keV}\right)$ to the $\mathrm{p}_{1 / 2}$ states at a continuum energy of $0.35 \mathrm{MeV}$. The case of a ${ }^{208} \mathrm{~Pb}$ target is considered.

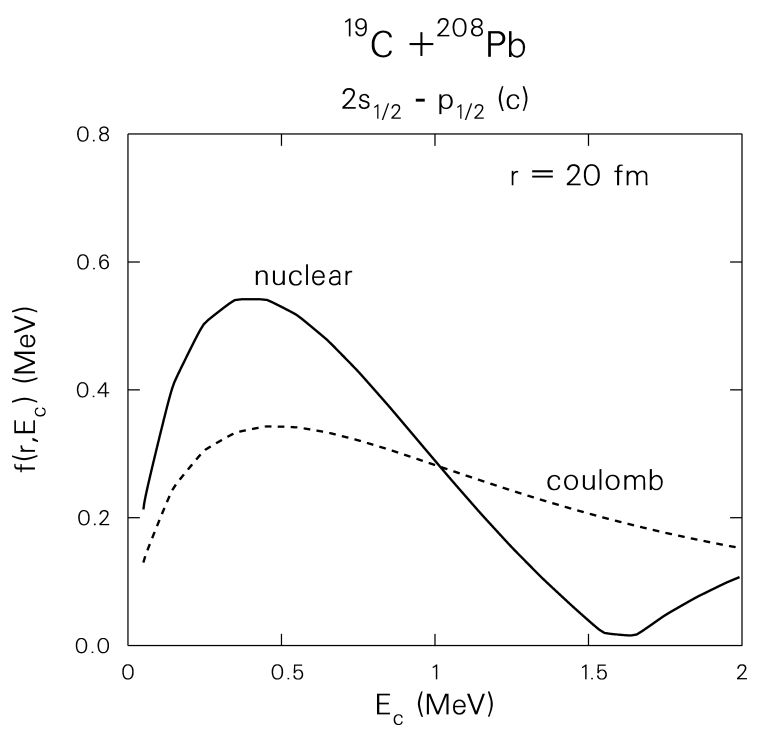

Fig. 3. Same as in Fig. 2, but as a function of the continuum energy, at a fixed distance of $20 \mathrm{fm}$.

than $15 \mathrm{fm}$. In a pure Rutherford trajectory this corresponds to an angle of about 2.5 degrees. Although the nuclear contribution is small in itself, the effect due to the interference is still quite large.

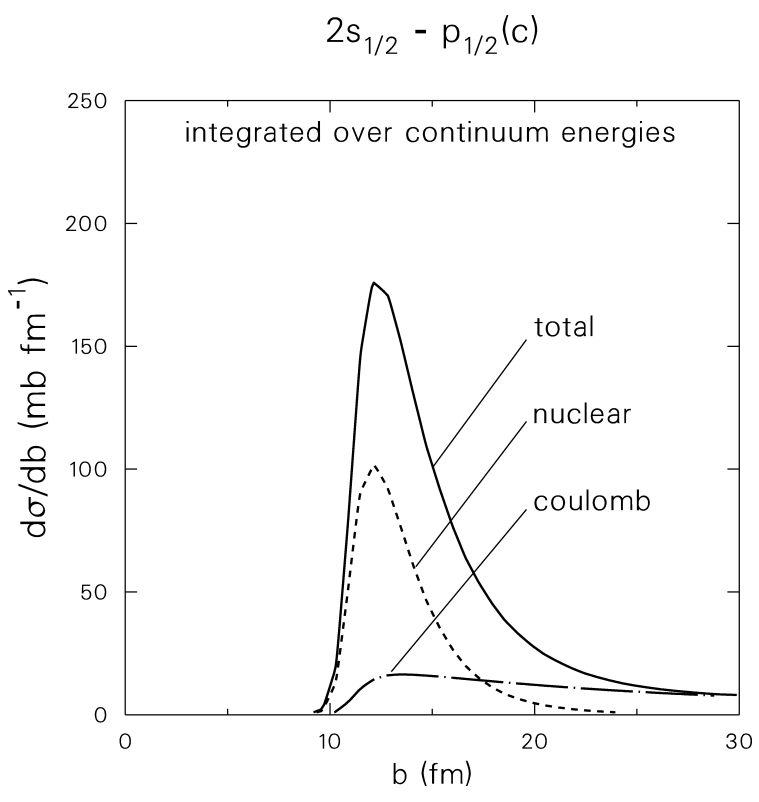

Fig. 4. Differential break-up cross sections as a function of the impact parameter. The calculation has been performed for the break-up of ${ }^{19} \mathrm{C}$ on a ${ }^{208} \mathrm{~Pb}$ target, at a bombarding energy of $E / A=67 \mathrm{MeV}$. The considered transition is from the bound $2 \mathrm{~s}_{1 / 2}$ state in ${ }^{19} \mathrm{C}\left(E_{b}=530 \mathrm{keV}\right)$ to all integrated $\mathrm{p}_{1 / 2}$ states in the continuum. The different curves refer to the case in which either the Coulomb or the nuclear break-up, or both, are considered.

We now consider the question of the target-mass dependence of the nuclear break-up cross section and show results for the systems ${ }^{19} \mathrm{C}+{ }^{12} \mathrm{C},{ }^{19} \mathrm{C}+{ }^{58} \mathrm{Ni}$ and ${ }^{19} \mathrm{C}+{ }^{208} \mathrm{~Pb}$. The differential break-up cross sections are given as a function of the impact parameter $b$ in the right panel of Fig. 1 The lowest figure shows the total cross section for the three targets ${ }^{12} \mathrm{C},{ }^{58} \mathrm{Ni}$ and ${ }^{208} \mathrm{~Pb}$, while the upper two figures show the corresponding results for the nuclear and Coulomb components. The values of the total cross sections are given close to each curve, showing again a large interference effect. Comparing with the left panel one can see that the nuclear contribution now exhibits a quite different dependence with the target mass. The total nuclear cross sections for the different targets, normalized to those of the lightest target considered, ${ }^{12} \mathrm{C}$, are plotted in Fig. 6 for both a "normal", or well bound, ${ }^{19} \mathrm{C}$ projectile and for the realistic case of a halo ${ }^{19} \mathrm{C}$ projectile. These values are compared in the figure with the predictions of scaling rules with $A^{1 / 3}$ and $A$, respectively. One can conclude 

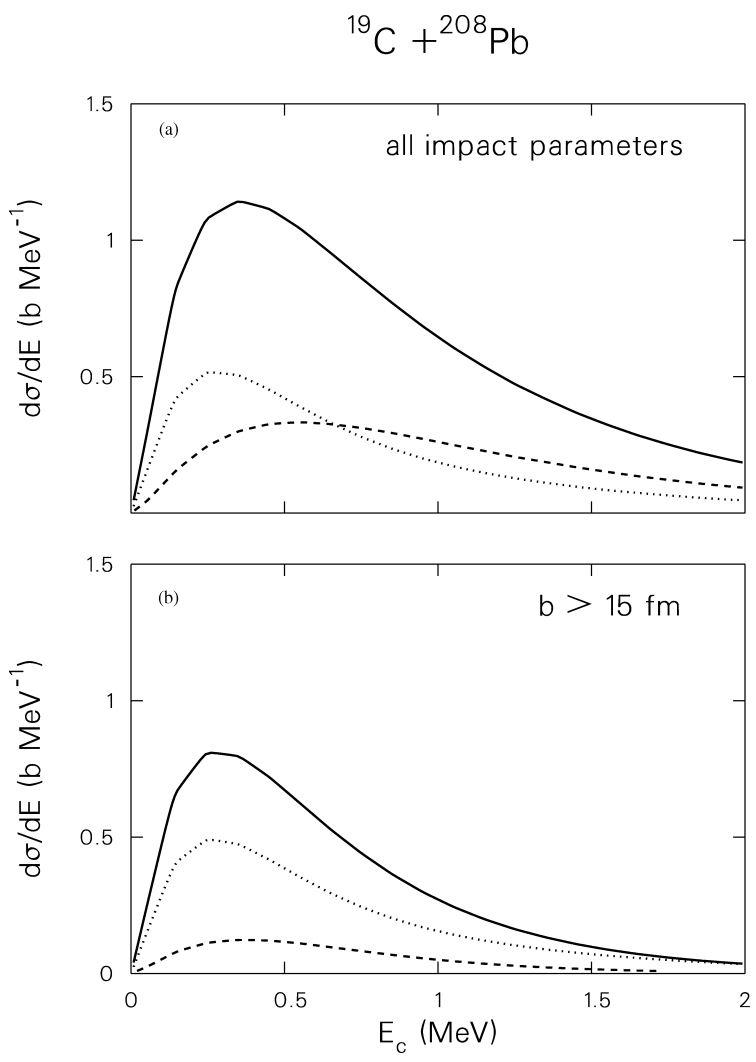

Fig. 5. Differential break-up cross sections as a function of the energy in the continuum. The calculation has been performed for the break-up of ${ }^{19} \mathrm{C}$ on a ${ }^{208} \mathrm{~Pb}$ target, at a bombarding energy of $E / A=67 \mathrm{MeV}$. The considered transition is from the bound $2 \mathrm{~s}_{1 / 2}$ state in ${ }^{19} \mathrm{C}\left(E_{b}=530 \mathrm{keV}\right)$ to $\mathrm{p}_{1 / 2}$ states in the continuum. The different curves refer to the case in which either the Coulomb or the nuclear break-up, or both, are considered. In (a) the contributions from all impact parameters are included, while in (b) only the peripheral impact parameters larger than $15 \mathrm{fm}$ have been considered.

that the total nuclear cross sections approximately scale linearly with the target mass, at variance with the approximately $A^{1 / 3}$ dependence obtained for a well bound projectile. This is a consequence of the extended transition density in the halo projectile and results in a behavior somewhat similar to the one associated with the Coulomb mechanism in ordinary nuclei.

In summary, we have shown that the nuclear breakup of halo nuclei displays features that are at variance with those characteristic of processes involving stable systems. In particular the long range of the nuclear

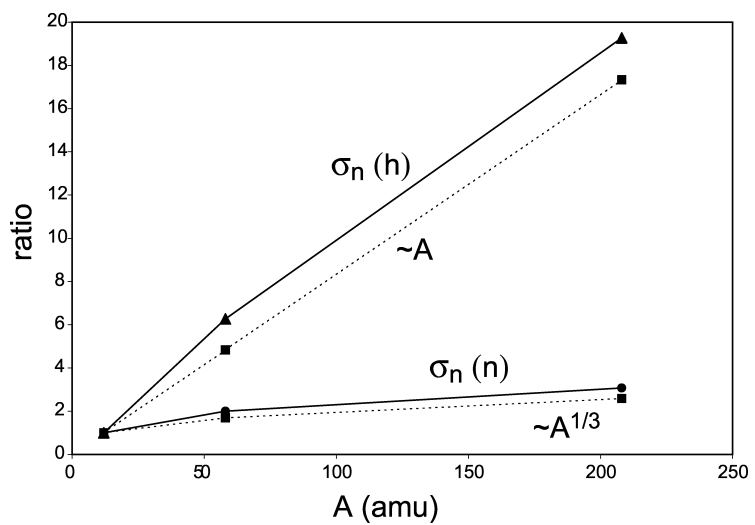

Fig. 6. Total nuclear cross sections for each target, normalized to those corresponding to the target ${ }^{12} \mathrm{C}$ as a function of the target mass (full lines) for both a well bound (n) and a halo (h) ${ }^{19} \mathrm{C}$ projectile. These curves are compared with the predictions of $A^{1 / 3}$ and $A$ scaling rules (dotted lines).

formfactors leads to a target-mass dependence of the nuclear cross section considerably stronger than the familiar $A^{1 / 3}$ scaling law and to a more effective Coulomb-nuclear interference.

\section{References}

[1] G. Baur, C.A. Bertulani, D.M. Kalassa, Nucl. Phys. A 550 (1992) 163

[2] T. Motobayashi et al., Phys. Rev. Lett. 73 (1994) 2680.

[3] H. Esbensen, G.F. Bertsch, Phys. Rev. C 59 (1999) 3240.

[4] F. Nunes, I.J. Thompson, Phys. Rev. C 59 (1999) 2652.

[5] F. Barranco, E. Vigezzi, R. Broglia, Phys. Lett. B 319 (1993) 387.

[6] E. Garrido, D.V. Fedorov, A.S. Jensen, Europhys. Lett. 50 (2000) 735 .

[7] D.L. Olson, B.L. Berman, D.E. Greiner, H.H. Heckman, P.J. Lindstrom, H.J. Crawford, Phys. Rev. C 28 (1983) 1602.

[8] R. Serber, Phys. Rev. 72 (1947).

[9] T. Nakamura et al., Phys. Lett. B 394 (1997) 11.

[10] T. Nakamura et al., Phys. Rev. Lett. 83 (1999) 1112.

[11] P. Banerjee, R. Shyam, Phys. Rev. C 61 (2000) 047301.

[12] P. Descouvemont, Nucl. Phys. A 675 (2000) 559.

[13] C.H. Dasso, S.M. Lenzi, A. Vitturi, Nucl. Phys. A 611 (1996) 124.

[14] C.H. Dasso, S.M. Lenzi, A. Vitturi, Nucl. Phys. A 639 (1998) 635.

[15] A. Bonaccorso, F. Cardtoiu, Phys. Rev. C 61 (2000) 034605.

[16] H. Esbensen, K. Henchen, Phys. Rev. C 61 (2000) 054606.

[17] O. Akyuz, A. Winther, in: R.A. Broglia, C.H. Dasso, R.A. Ricci (Eds.), Proceedings of the Enrico Fermi International School of Physics, 1979, Course on Nuclear Structure and Heavy Ion Reactions, North-Holland, Amsterdam, 1981. 